International Mathematical Forum, 1, 2006, no. 5, 237-255

\title{
Techniques for a priori Choice of Regularizing Parameters in Tikhonov Regularization
}

\author{
M. Iqbal \\ Department of Mathematical Sciences \\ King Fahd University of Petroleum and Minerals \\ Dhahran 31261, Saudi Arabia \\ miqbal@kfupm.edu.sa
}

\begin{abstract}
In this paper, we propose a new strategy for a priori choice of regularization parameters in Tikhonov's regularization, based on the conditional stability estimate for ill-posed inverse problems. We show that it can be applied to a wide class of inverse problems. The convergence rate of the regularized solutions is also proved.
\end{abstract}

Mathematics Subject Classification: 65R20, 65R30

Keywords: Ill-posed problems, convolution equation, Turchin's method, cross-validation, filter function, a-priori, a posteriori, periodogram, regularization parameter, Plancherel's theorem, Bayes' theorem.

\section{Introduction.}

We consider a solution $f$ of an operator equation

$$
K f=g
$$

Let $X, Y$ be Banach spaces and the operator $K: X \rightarrow Y$ is densely defined and is injective. We do not assume the continuity of $K$. The operator $K$ may also be nonlinear. Many types of inverse problems, such as identification 
of coefficients in partial differential equations, are described by this kind of operator equation $K f=g$.

We assume that $K^{-1}$ is not continuous from $Y$ to $X$ or that the range $R(K)$ is not closed in $Y$, which causes the ill-posed problem in solving $f$ in $K f=g$.

In modern science there is an increasingly important class of inverse problems which are not amenable to classical statistical estimation procedures and such problems are termed ill-posed. The notion of ill-posedness is usually attributed to Hadamard. Then, for stable reconstruction of $f$, some regularization techniques are necessary. The Tikhonov regularization techniques are widely applicable; for example, Bakushinsky and Goncharsky [2], Beuneister [3], Engle [9], Groetsch [12], Hofmann [13], Lavrent'v et al [16], Tikhonov and Arsenin [28], Tikhonov et al [29], Vasin and Ageev [32], Varah [31], Wahba [33], Brianzi [5], Essah and Delves [10], Ang [1], Gelfat [11], Eggermont [8], Bertero [4], Pinkus [22], and Bruckner [6]. For detailed bibliography, the reader should consult Piessens [20], Piessens and Branders [21]. A review and comparison is given in Davies [7], Talbot [25], Istratov [14], Shan [23] and Mead [17].

Schematically, the Tikhonov regularization can be stated as follows:

For given data $g$ in (1.1), we search for a minimizer of a functional

$$
C(f ; \lambda)=\|K f-g\|_{2}^{2}+\lambda\left\|f^{(p)}\right\|_{2}^{2}
$$

which is minimized over the subspace $H^{(p)} \subset L_{2}$.

Both norms in (1.2) are $L_{2}, f^{(p)}$ denotes the $p$-th derivative of $f$ and $\lambda>0$ is the regularization parameter.

The minimizer of (1.2) in $H^{p}$ is given by

$$
f_{\lambda}(y)=\frac{1}{2 \pi} \int_{-\infty}^{\infty} z(\omega ; \lambda) \frac{\widehat{g}(\omega)}{\widehat{k}(\omega)} \exp (i \omega y) d \omega
$$

where $z(\omega ; \lambda)$ is the filter function given by

$$
z(w ; \lambda)=\frac{|\widehat{k}(\omega)|^{2}}{|\widehat{k}(\omega)|^{2}+\lambda \omega^{2 p}} .
$$

We assume throughout that the support of each function $f, g$ and $k$ is essentially finite and contained within the interval $[0,1)$, possibly by a change of 
variable. It is then convenient to adopt the approximating function space $T_{N}$ of trigonometric polynomials of degree at most $N$ and period 1 , since then the disrcetization error in the convolution may be made exactly zero at the grid points and fast Fourier transforms (FFTs) may be employed in the solution procedure.

Let $g$ and $k$ be given at $N$ equally spaced points $x_{n}=n h, \quad n=0,1,2, \ldots, N-1$ with spacing $=h=1 / N$. Then $g$ and $k$ are interpolated by $g_{N}$ and $k_{N} \in T_{N}$, where

$$
g_{N}(x)=\frac{1}{N} \sum_{q=3 D 0}^{N-1} \widehat{g}_{N, q} \exp \left(i \omega_{q} x\right)
$$

and

$$
\widehat{g}_{N, q}=\sum_{n=3 D 0}^{N-1} g_{n} \exp \left(-i \omega_{q} x_{n}\right)
$$

where

$$
g\left(x_{n}\right)=g_{n}=g_{N}\left(x_{n}\right), \quad \omega_{q}=2 \pi q
$$

with similar expressions for $K_{N}$.

If equation (1.2) is now replaced by

$$
\left(K_{N} f_{N}\right)(x)=\int_{0}^{1} K_{N}(x-y) f_{N}(y) d y=g_{N}(x)
$$

where $K_{N}$ is periodically continued outside [0,1), then we can prove that the discretization error in the convolution is precisely zero at the grid points $\left\{x_{n}\right\}$.

Since $\underline{g}=\left(g_{n}\right)$ interpolates $g_{N}(x)$ in (1.8) at the grid points, it follows that the discretization error in the convolution vanishes here.

In $T_{N}, f_{\lambda}$ in (1.3) is approximated by

$$
f_{N ; \lambda}(x)=\sum_{q=3 D 0}^{N-1} z_{q ; \lambda} \frac{\widehat{g}_{N, q}}{\widehat{k}_{N, q}} \exp \left(i \omega_{q} x\right)
$$

where $z_{q ; \lambda}$ is the discrete $p$-th order filter given by

$$
z_{q ; \lambda}=\frac{\left|\widehat{k}_{N, q}\right|^{2}}{\left|\widehat{k}_{N, q}\right|^{2}+N^{2} \lambda \tilde{\omega}_{q}^{2 p}}
$$


where

$$
\tilde{\omega}_{q}= \begin{cases}\omega_{q}, & 0 \leq q<\frac{1}{2} N \\ \omega_{N-q}, & \frac{1}{2} N \leq q \leq N-1\end{cases}
$$

The optimal $\lambda$ in (1.10) is still to be determined and in next sections we discuss how to close it.

\section{Determination of Regularization Parameter $(\lambda)$}

\section{a) Turchin-Klein Method (TK).}

An ensemble which describes the fidelity of the random vector $K f$ to the data $\underline{g}$ and which is characterized by an a priori multivariate normal distribution with conditional density function (c.d.f.) $P(\underline{g} / \underline{f})$. Now an ensemble of smooth functions consistent with $p$-th order regularization and characterized by an a priori probability density function (p.d.f.) $P_{\lambda}(\underline{f})$, Turchin [30]. The filtered solution (1.9) is then the mathematical expectation is then has an $a$ posteriori (c.d.f.) given by Bayes' theorem $P(\underline{f} / \underline{g})=\operatorname{constant} P(\underline{g} / f) P_{\lambda}(\underline{f})$, (see Klein [15]).

The optimal $\lambda$ is defined as that value, which makes the variance of the $a$ posterior ensemble equal to the variance $\sigma^{2}$. The regularization parameter $\lambda$ is determined by solving the non-linear equation

$$
\frac{\sigma^{2}}{N} \sum_{q=0}^{N-1} z_{q ; \lambda}+\sum_{q=0}^{N-1}\left|\widehat{g}_{N, q}\right|^{2}\left(1-z_{q ; \lambda}\right)^{2}=\sigma^{2} .
$$

\section{b) Wahba's Cross-validation [33] Method}

From equation (1.9) we know that the filtered solution $f_{N, \lambda}(x) \in T_{n}$ which minimizes

$$
\sum_{n=0}^{N-1}\left[\left(K_{N} * f\right)\left(x_{n}\right)-g_{n}\right]^{2}+\lambda\left\|f^{(p)}(x)\right\|_{2}^{2}
$$

is

$$
f_{N ; \lambda}(x)=\frac{1}{N} \sum_{q=0}^{N-1} \widehat{f}_{N, \lambda, q} \exp (2 \pi i q x)
$$


where $\widehat{f}_{N, \lambda, q}=z_{q ; \lambda} \frac{\widehat{g}_{N, q}}{\widehat{k}_{N, q}}$ with $z_{q ; \lambda}$ is given in equation (1.10).

The idea of generalized cross-validation (GCV) [33], is quite simple to understand. Suppose we ignore the $j$-th data point $g_{j}$ and define the filtered solution $f_{N, \lambda}^{[j]}(x) \in T_{N}$ as the minimizer of

$$
\sum_{\substack{n=0 \\ n \neq j}}^{N-1}\left[\left(K_{N} * f\right)\left(x_{n}\right)-g_{n}\right]^{2}+\lambda\left\|f^{(p)}(x)\right\|_{2}^{2} .
$$

Then we get a vector $\underline{g}_{N, \lambda}^{[j]} \in \mathbb{R}$ defined by

$$
\underline{g}_{N, \lambda}^{[j]}=K \underline{f}_{N, \lambda}^{[j]} .
$$

Clearly, the $j$-th element $g_{N, \lambda, j}^{[j]}$ of equation (3.3) should 'predict' the missing value $g_{j}$.

We may thus construct the weighted mean square prediction error over all $j$ :

$$
V(\lambda, p)=\frac{1}{N} \sum_{j=0}^{N-1} \omega_{j}(\lambda)\left(g_{N, \lambda, j}^{[j]}-g_{j}\right)^{2}
$$

The principle of GCV applied to the deconvolution problem then says that the best filtered solution to the problem should minimize (2.4). Thus the optimal $\lambda$ minimizes $V(\lambda, p)$ for given $p$ and does not require the knowledge of $\sigma^{2}$.

To minimize $V(\lambda, p)$ in $(2.4)$ is a time-consuming problem. Wahba has suggested an alternative expression which depends on a particular choice of weights, resulting in considerable simplification. Let

$$
\underline{f}_{N, \lambda}=\left(f_{N, \lambda}\left(x_{0}\right), \ldots, f_{N, \lambda}\left(x_{N-1}\right)\right)^{T}
$$

and define

$$
\underline{g}_{N, \lambda}=K \underline{f}_{N, \lambda} .
$$

Then there exists a matrix $A(\lambda)$, called an influence matrix, such that

$$
\underline{g}_{N, \lambda}=A(\lambda) \underline{g}_{N} .
$$


Let $\widehat{k}=\operatorname{diag}\left(h K_{N, q}\right)$ and $\widehat{z}=\operatorname{diag}\left(z_{q ; \lambda}\right)$. Then from (1.9), we see that

$$
\underline{f}_{N, \lambda}=\psi \widehat{k}^{-1} \widehat{z}_{N}
$$

where $\psi$ is the unitary matrix with elements

$$
\psi_{r s}=\frac{1}{\sqrt{N}} \exp \left(\frac{2 \pi}{N} i r s\right), \quad r, s=0,1, \ldots, N-1
$$

and has the property $(K \underline{f})_{n}=\left(K_{N} f\right)\left(x_{n}\right)$. Also, $\widehat{g}_{N}=\psi^{H} g_{N}$ and so

$$
A(\lambda)=\psi \widehat{z} \psi^{H}
$$

since

$$
K=\psi \widehat{K} \psi^{H}
$$

Wahba [33] has shown, in a more general context, that the choice of weights

$$
\omega_{j}(\lambda)=\left[\frac{1-a_{j j}(\lambda)}{\frac{1}{N} \operatorname{Trace}(1-A(\lambda))}\right]^{2}, \quad j=0,1, \ldots, N-1,
$$

where $A(\lambda)$ is the influence matrix in equation (2.7), enables the expression (2.4) to be written in the simpler form

$$
V(\lambda, p)=\frac{\frac{1}{N} \|\left(I-A\left(\lambda() \underline{g}_{N} \|_{2}^{2}\right.\right.}{\left[\frac{1}{N} \operatorname{Trace}(I-A(\lambda))\right]^{2}} .
$$

Using equation (2.8) it follows that

$$
V(\lambda, p)=\frac{\frac{1}{N}\left\|(I-\widehat{z}) \widehat{g}_{N}\right\|_{2}^{2}}{\left[\frac{1}{N} \operatorname{Trace}(I-\widehat{z})\right]^{2}}
$$

i.e.,

$$
V(\lambda, p)=\frac{\frac{1}{N} \sum_{q=0}^{N-1}\left(1-z_{q, \lambda}\right)^{2}\left|\widehat{g}_{N, q}\right|^{2}}{\left[\frac{1}{N} \sum_{q=0}^{N-1}\left(1-z_{q ; \lambda}\right)\right]^{2}} .
$$

Since the matrix in (2.8) is circulant, the weights in (2.9) are all unity. The optimal value of $\lambda$ is determined by minimizing $V(\lambda, p)$ in equation (2.11). 


\section{c) Bayesian Method}

\section{The filter in a stochastic setting}

Here we relate the $p$-th order convolution filter (1.10) to certain spectral densities which play a role in the optimization of $\lambda$. Assume that the data $\left\{g_{n}\right\}$ are noisy and that there is an underlying function $U_{n} \in T_{N}$ such that

$$
g_{n}=u_{N}\left(x_{n}\right)+\epsilon_{n} \equiv u_{n}+\epsilon_{n}
$$

We identify both $\left\{u_{n}\right\}$ and $\left\{\epsilon_{n}\right\}$ with independent stationary stochastic processes. Since, in general, the expectation $E\left(u_{n}\right)$ is not zero, it is suggested by Mead [17], Yamanoto [35], and Thompson [26, 27], that the data $\left\{g_{n}\right\}$ be detrended so that $U_{n}$ becomes weakly stationary. This would involve subtracting from the data the values of a smooth function of roughly the same shape as $u_{N}$.

In this paper, we do not detrend. In the limit $N \rightarrow \infty, h \rightarrow 0$ for any discrete process $X_{n}$ we may write

$$
X_{n}=\int_{0}^{1} \exp (2 \pi i \omega n) d S_{X}(\omega)
$$

where $S_{X}(\omega)$ is a stochastic process defined on $[0,1)$. The essential property of $S_{X}$ we require is given below,

Lemma 1. The variance of any integral $\int \theta(\omega) d S_{X}(\omega)$ is given by $\int|\theta(\omega)|^{2} d S_{X}(\omega)$ where $d S_{X}(\omega)=3 D=E\left(\left|d S_{X}(\omega)\right|^{2}\right)$.

Proof. $G_{X}(\omega)$ may be interpreted as a spectral distribution function and accordingly we shall write $d S_{X}(\omega)=P_{X}(\omega) d \omega$ where $P_{X}(\omega)$ is a spectral density.

Now consider $f_{N} \in T_{N}$ with

$$
\underline{f}=\left\{f_{n}\right\} \equiv f_{N}\left(x_{n}\right)
$$

defined by $(K \underline{f})_{n}=u_{n}, n=0,1, \ldots, N-1$, where $K$ is given by equation 
(2.9). From (2.13), we have

$$
\begin{aligned}
f_{n} & =\sum_{m=0}^{N-1}\left\{\left(K^{-1}\right)_{m n} \int_{0}^{1} \exp (2 \pi i \omega n) d S_{u}(\omega)\right\} \\
& =\int_{0}^{1}[\widehat{K}(\omega)]^{-1} \exp (2 \pi i \omega n) d S_{u}(\omega)
\end{aligned}
$$

where

$$
\widehat{K}_{N}(\omega)=\sum_{n=0}^{N-1} K_{n} \exp (-2 \pi i \omega n)
$$

Assume that $f_{n}$ is estimated by $\sum_{m=0}^{N-1} \ell_{m} g_{n-m}$ where $\left\{\ell_{m}\right\}$ is a filter which we shall relate to $z_{q ; \lambda}$ and $\left\{g_{n}\right\}$ is periodically continued for $n \notin[0, n)$. Then the error

$$
f_{n}-\sum_{m=0}^{N-1} \ell_{m} g_{n-m}
$$

is given by

$$
\int_{0}^{1} \exp (2 \pi i \omega n)\left([\widehat{k}(\omega)]^{-1}-\widehat{\ell}_{N}(\omega) d S_{u}(\omega)\right)-\int_{0}^{1} \exp (2 \pi i \omega n) \widehat{\ell}_{N}(\omega) d S_{\epsilon}(\omega)
$$

were $\ell_{N}(\omega)$ is defined as in equation (2.14).

The variance of the error is clearly

$$
\int_{0}^{1}\left|\left[\widehat{k}_{\omega}\right]^{-2}-\widehat{\ell}_{N}(\omega)\right|^{2} P_{u}(\omega) d \omega+\int_{0}^{1}\left|\widehat{\ell}_{N}(\omega)\right|^{2} P_{\epsilon}(\omega) d \omega
$$

which is minimized when

$$
\widehat{\ell}_{N}(\omega) \widehat{k}_{N}(\omega)=P_{u}(\omega) /\left(P_{u}(\omega)+P_{\epsilon}(\omega)\right)
$$

Since the discrete Fourier coefficients of the filtered solution must satisfy $\widehat{f}_{N, q ; \lambda}=h \widehat{\ell}_{N, q} \widehat{g}_{N, q}=z_{q ; \lambda} \widehat{g}_{N, q}\left[h \widehat{K}_{N, q}\right]^{-1}$, we find $z_{q ; \lambda}=h^{2} \widehat{\ell}_{N, q} \widehat{K}_{N, q}$. Thus, from the observation $h \widehat{\ell}_{N, q}=\widehat{\ell}_{N}(q h), h \widehat{k}_{N, q}=\widehat{k}(q h)$, we have from (2.18) the following theorem. 
Theorem. In the limit $N \rightarrow \infty, h \rightarrow 0$, the variance of the error $f_{N}\left(x_{n}\right)-$ $f_{N ; \lambda}\left(x_{n}\right)$ is minimized at $x_{n}$ by the choice of filter

$$
z_{q ; \lambda}=\frac{P_{u}(q h)}{P_{u}(q h)+P_{\epsilon}(q h)} .
$$

Proof. We now simply relate the filter in (2.19) to the $p$-th order filter in (1.10). Assuming that the errors are uncorrelated, $P_{\epsilon}(\omega)$ has the form

$$
P_{\epsilon}(\omega)=\sigma^{2}=\text { constant }
$$

where $\sigma^{2}$ is the unknown variance of the noise in the data. Choosing

$$
P_{u}(\omega)=\frac{\sigma^{2}\left|\widehat{k}_{N}(\omega)\right|^{2}}{\lambda \tilde{\omega}^{2 p}}
$$

where

$$
\tilde{\omega}= \begin{cases}2 \pi N w, & 0 \leq \omega<\frac{1}{2} \\ 2 \pi N(1-\omega), & \frac{1}{2} \leq \omega<1 .\end{cases}
$$

Then we get (1.9) from equation (2.19). Moreover, the spectral density for $\left\{g_{n}\right\}$ is then

$$
P_{g}(\omega)=P_{u}(\omega)+P_{\epsilon}(\omega)=\sigma^{2}\left[1+\frac{\left|\widehat{k}_{N}(\omega)\right|^{2}}{\lambda \tilde{\omega}^{2 p}}\right]
$$

where $P_{g}(q h)=\sigma^{2}\left(1-z_{q ; \lambda}\right)^{-1}$.

The statistical likelihood of any suggested values of $\sigma^{2}$ and $\lambda$ may now be estimated from the data. Following Whittle [34] and Thompson [27], the logarithm of the likelihood function of $P_{g}$ is given approximately by (Whittle $[34])$,

$$
\text { const }-\frac{1}{2} \sum_{q=0}^{N-1}\left[\log P_{g}(q h)+I(q h) / P_{g}(q h)\right]
$$

where $I(\omega)=\left|\sum_{n=0}^{N-1} g_{n} \exp (-2 \pi i \omega n)\right|^{2}$ is the periodogram of the data with $I(q h)=\left|\widehat{g}_{N, q}\right|^{2}$. 
We now maximize (2.22) with respect to $\sigma^{2}$ and $\lambda$. The partial maximum with respect to $\sigma^{2}$ may be found exactly (in terms of $\lambda$ ) with the maximizing value of $\sigma^{2}$ given by

$$
\sigma^{2}=\frac{1}{N} \sum_{q=1}^{N-1}\left|\widehat{g}_{N, q}\right|^{2}\left(1-z_{q ; \lambda}\right)
$$

The maximum with respect to $\lambda$ may then be found by minimizing

$$
V(\lambda)=\frac{1}{2} N \log \left[\sum_{q=1}^{N-1}\left|\widehat{g}_{N, q}\right|^{2}\left(1-z_{q ; \lambda}\right)\right]-\frac{1}{2} \sum_{q=1}^{N-1} \log \left(1-z_{q ; \lambda}\right) .
$$

Thus the optimal value of the regularization parameter $\lambda$ is given by the minimizer of (2.24), which is a simple function of $\lambda$, depending upon the known Fourier coefficients $\widehat{g}_{N, q}$ and $\widehat{k}_{N, q}$. No prior knowledge of $\sigma^{2}$ is assumed but $a$ posteriori estimate is given by equation (2.23).

\section{Error Analysis}

The order of convergence for trigonometric regularization is described in Theorem below. The proof requires the simple observation in Lemma 2.

Convergence of regularized solutions starting from data polluted with errors is an important issue. More precisely, when $K f=g$ where $g$ is a data without noise. Assuming a noise level $\epsilon>0:\left\|g_{\epsilon}-g\right\|<\epsilon$, we have to reconstruct a stable approximation (Natterer [19] and Baumeister [3]), to $f$ by $g_{\epsilon}$.

Lemma 2. If the periodic function $f$ has an absolutely continuous $r$-th derivative $f^{(r)}$, then its exact Fourier coefficients $\widehat{f}_{q}$ satisfy

$$
\left|\widehat{f}_{q}\right|=0\left[\frac{1}{|q|^{r+1}}\right]
$$

The proof is given in Stoer and Bulirsch [24]. 
Theorem. If $f \in C^{P}(0,1)$, then

$$
\begin{aligned}
E\left(\left\|f-f_{N ; \lambda}\right\|^{2}\right)= & 0\left(h^{2 p}\right)+\lambda^{2} \sum_{q=0}^{N-1}\left[\frac{\tilde{\omega}_{q}^{4 p}\left|\widehat{f}_{N, q}\right|^{2}}{\left(\left|k_{N, q}\right|^{2}+\lambda N^{2} \tilde{\omega}_{q}^{2 p}\right)^{2}}\right] \\
& +N \sigma^{2} \sum_{q=0}^{N-1}\left[\frac{\left|\widehat{k}_{N, q}\right|^{2}}{\left(\left|\widehat{k}_{N, q}\right|^{2}+N^{2} \lambda \tilde{\omega}_{q}^{2 p}\right)^{2}}\right] .
\end{aligned}
$$

Proof. Let $f_{N ; \lambda}^{0}$ be the filtered solution for exact data with given value of $\lambda$ and $f_{N ; \lambda}$ be the filtered solution with inexact (noisy) data. The total error may be written as

$$
f-f_{N ; \lambda}=\left(f-f_{N}\right)+\left(f_{N}-f_{N ; \lambda}^{0}\right)+\left(f_{N ; \lambda}^{0}-f_{N ; \lambda}\right)
$$

where the three terms on the right hand side of (3.2) represent the aliasing error (projection onto $T_{N}$ ), the regularization error and the error resulting from the noise in the data respectively.

Assuming that these three sources of errors are mutually independent, we then have

$$
E\left(\left\|f-f_{N ; \lambda}\right\|^{2}\right)=E\left(\left\|f-f_{N}\right\|^{2}\right)+E\left(\left\|f_{N}-f_{N ; \lambda}^{0}\right\|^{2}\right)+E\left(\left\|f_{N ; \lambda}^{0}-f_{N ; \lambda}\right\|^{2}\right) .
$$

Let $\widehat{f}_{q}$ denote the exact Fourier coefficients of $f$ on $[0,1)$; then $f(x) \sim$ $\frac{1}{N} \sum_{q=-\infty}^{\infty} \widehat{f}_{q} \exp \left(i \omega_{q} x\right)$ where $\left|\widehat{f}_{q}\right|=0(N q)^{-p}$ by equation (3.1). Using Hamming's analysis of aliasing, we have $\widehat{f}_{N, q}=\widehat{f}_{q}+\sum_{r=1}^{\infty}\left(\bar{f}_{N r-q}+\widehat{f}_{N r+q}\right)$. Thus for the first term on the right of (3.3) we have

$$
\begin{aligned}
\left\|f-f_{N}\right\|^{2} & =\frac{1}{N^{2}}\left[\sum_{q=-\frac{1}{2} N+1}^{\frac{1}{2} N-1}\left|\widehat{f}_{q}-\widehat{f}_{N, q}\right|^{2}+2 \sum_{q=\frac{1}{2} N}^{\infty}\left|\widehat{f}_{q}\right|^{2}\right] \\
& =0\left(N^{-2 p}\right)=0\left(h^{2 p}\right) .
\end{aligned}
$$

For the second term on the right hand side of (3.3), using Plancehrel's theorem, we have

$$
\left\|f_{N}-f_{N ; \lambda}^{0}\right\|^{2}=\frac{1}{N^{2}} \sum_{q=0}^{N-1}\left(1-z_{q ; \lambda}\right)^{2}\left|\widehat{f}_{N, q}\right|^{2}=\lambda^{2} \sum_{q=0}^{N-1}\left[\frac{\tilde{\omega}_{q}^{4 p}\left|\widehat{f}_{N ; q}\right|^{2}}{\left[\left|\widehat{K}_{N, q}\right|^{2}+N^{2} \lambda \tilde{\omega}_{q}^{2 p}\right]^{2}}\right]
$$


For the third term on the right hand side of (3.3)

$$
E\left(\left\|f_{N ; \lambda}^{0}-f_{N ; \lambda}\right\|^{2}\right)=E\left[\sum_{q=0}^{N-1} z_{q ; \lambda}^{2} \frac{\left|\widehat{h}_{\epsilon_{N, q}}\right|^{2}}{\left|\widehat{k}_{N, q}\right|^{2}}\right]=N \sigma^{2} \sum_{q=0}^{N-1}\left[\frac{z_{q ; \lambda}^{2}}{\left|\widehat{k}_{N, q}\right|^{2}}\right] .
$$

Since

$$
\begin{aligned}
\sigma^{2} & =E\left(\epsilon_{n}^{2}\right) \\
& =\frac{1}{N} E\left(\left|\widehat{\epsilon}_{N, q}\right|^{2}\right)
\end{aligned}
$$

putting values we get the theorem immediately.

\section{Addition of Random Noise to the Data Functions}

In solving the ill-posed problems, which we shall discuss in the next section, we have considered the data functions contaminated by varying amounts of random noise. To generate sequences of random errors of the form $\left\{\epsilon_{n}\right\}, n=$ $0,1, \ldots, N-1$, we have used the NAG Algorithm G05DAA which returns pseudo-random real numbers taken from a normal distribution of prescribed mean A and standard deviation B. To mimic experimental errors, we have

$$
A=0 \text { and } B=\frac{X}{100}\left(\max _{0 \leq n \leq N-1}\left\{g_{n}\right\}\right)
$$

where $X$ denotes a chosen percentage, e.g. $X=0.7,1.7,3.3$ and 6.7. Thus the random error $\epsilon_{n}$ added to $g_{n}$ does not exceed $3 X \%$ of the maximum value of $g(x)$.

\section{Numerical Examples}

Example 1. This example has been taken from Turchin [30].

$$
\int_{-2}^{2} K(x-y) f(y) d y=g(x)
$$

where $f$ is the sum of two Gaussian functions

$$
f(x)=0.5 \exp \left[-\frac{(x+0.4)^{2}}{0.18}\right]+\exp \left[-\frac{(x-0.6)^{2}}{0.18}\right]
$$


and $K(x)$ is triangular with equation

$$
K(x)=\left[\begin{array}{cc}
(5 / 12)(-x+1.2), & 0 \leq x<1.2 \\
(5 / 12)(x+1.2), & -1.2 \leq x<0 \\
0, & |x| \geq 1.2 .
\end{array}\right.
$$

The essential support of $g(x)$ is $-2.5<x<2.7$.

Example 2. This example has been taken from Medgyessy [18]. The solution function is the sum of six Gaussians and the kernel is also Gaussian.

$$
\int_{-\infty}^{\infty} k(x-y) f(y) d y=g(x)
$$

where $g(x)=\sum_{k=1}^{6} A_{k} \exp \left[\frac{\left(x-a_{k}\right)^{2}}{b_{k}}\right]$,

$$
\begin{array}{lll}
A_{1}=10 & a_{1}=0.5 & b_{1}=0.04 \\
A_{2}=10 & a_{2}=0.7 & b_{2}=0.02 \\
A_{3}=5 & a_{3}=0.875 & b_{3}=0.02 \\
A_{4}=10 & a_{4}=1.125 & b_{4}=0.04 \\
A_{5}=5 & a_{5}=1.325 & b_{5}=0.02 \\
A_{6}=5 & a_{6}=1.525 & b_{6}=0.02
\end{array}
$$

The essential support of $g(x)$ is $0<x<2$. The kernel is

$$
k(x)=\frac{1}{\sqrt{\pi \lambda}} \exp \left(-\frac{x^{2}}{\lambda}\right), \quad \lambda=0.015
$$

with essential support $(-0.26,0.26)$.

The solution function is

$$
f(x)=\sum_{k=3 D 1}^{6}\left\{\left[\frac{b_{k}}{b_{k}-\lambda}\right]^{1 / 2} A_{k} \exp \left[-\frac{\left(x-a_{k}\right)^{2}}{\left(b_{k}-\lambda\right)}\right]\right\}
$$


with essential support $(0.26,1.74)$.

\section{Numerical Solutions}

In this section we describe the numerical results which we have obtained for each example and for each method. Throughout we use second order regularization exclusively, i.e., $p=2$ and the number of data points $N=64$.

a) TK Method (Turchin-Klein Method)

The results are shown in Table 1.

Table 1

\begin{tabular}{|c|c|c|c|c|}
\hline Examples & $N$ & Noise level & $\sigma$ & $\left\|f-f_{N}\right\|_{2}$ \\
\hline Example 1 & 64 & $0.0 \%$ & $2.20 \times 10^{-6}$ & $2.20 \times 10^{-2}$ \\
\hline & 64 & $0.7 \%$ & $2.42 \times 10^{-3}$ & $1.77 \times 10^{-1}$ \\
\hline Example 2 & 64 & $0.0 \%$ & $1.83 \times 10^{-2}$ & $2.57 \times 10^{-1}$ \\
\hline & 64 & $1.7 \%$ & $1.89 \times 10^{-1}$ & $3.52 \times 10^{0}$ \\
\hline
\end{tabular}

\section{b) Cross-validation Method}

Example 1 is a highly ill-posed problem. For accurate data the method yields perfect solution, resolving the two peaks very clearly but with $0.7 \%$ noise it does not resolve the peaks very clearly. The results are shown in Table 2 .

Example 2 For accurate data the results are very good but with 1.7\% noise it is reasonably good and results are shown in Table 2 . 
Table 2

\begin{tabular}{|c|c|c|c|c|}
\hline Examples & $N$ & Noise level & $\lambda$ & $\left\|f-f_{N}\right\|_{2}$ \\
\hline Example 1 & 64 & $0.0 \%$ & $4.30 \times 10^{-14}$ & $2.67 \times 10^{-3}$ \\
\hline & 64 & $0.7 \%$ & $1.631 \times 10^{-7}$ & $1.397 \times 10^{-1}$ \\
\hline Example 2 & 64 & $0.0 \%$ & $1.00 \times 10^{-19}$ & $9.30 \times 10^{-2}$ \\
\hline, & 64 & $1.7 \%$ & $1.60 \times 10^{-11}$ & $1.405 \times 10^{0}$ \\
\hline
\end{tabular}

\section{c) Bayesian Method}

As above the results are shown for both examples in Table 3.

Table 3

\begin{tabular}{|c|c|c|c|c|}
\hline Examples & $N$ & Noise level & $\lambda$ & $\left\|f-f_{N}\right\|_{2}$ \\
\hline Example 1 & 64 & $0.0 \%$ & $3.20 \times 10^{-16}$ & $7.25 \times 10^{-3}$ \\
\hline & 64 & $0.7 \%$ & $5.501 \times 10^{-11}$ & $2.301 \times 10^{-1}$ \\
\hline Example 2 & 64 & $0.0 \%$ & $3.40 \times 10^{-15}$ & $6.260 t i 10^{-1}$ \\
\hline, & 64 & $1.7 \%$ & $1.10 \times 10^{-11}$ & $3.545 \times 10^{0}$ \\
\hline
\end{tabular}




\section{Concluding Remarks}

All the three methods worked very well over both the test examples, which are severely ill-posed and the results are shown in Tables 1, 2 and 3 respectively. For the comparison purposes cross-validation method has slight edge over the other two methods and it yields slightly better results and is little less time consuming.

Recently such research has been developed enough so that we can emphasize the wide applicability of these methods in real inverse problems including integral transforms, e.g., Laplace transforms and Mellin transforms inversion.

Acknowledgment. The author gratefully acknowledges the excellent research and computer facilities availed at King Fahd University of Petroleum \& Minerals, Dhahran during the preparation of this paper.

\section{References}

1. Ang, D.D. et al, 'Complex variable and regularization methods of inversion of the Laplace transform', Journal of Mathematics of Computation, Vol. 53, Number 188(1989), pp. 589-608.

2. Bakushinsky, A and Goncharsky, A., 'Ill-posed Problems: Theory and applications' (1994), Published by Dordrecht Kluwer.

3. Bauneister, J., 'Stable solutions of Inverse Problems', (1987) (Braunschweig, Vieweg).

4. Bertero, M. and Pike, E.R., 'Exponential sampling methods for Laplace and other diagonally invariant transforms', J. Inverse Problems Vol. 7(1991), pp. 21-32.

5. Brianzi, P. and Frontini, M. 'On the regularized inversion of the Laplace transform' Inverse Problems 7 (1991) pp. 355-368.

6. Bruckner, G. and Cheng, J., 'Tikhonov regularization for an integral equation of the first kind with logarithmic kernel', J. Inverse and Illposed Problems (2000), pp. 21-33. 
7. Davies, B. and Martin, B. 'Numerical inversion of the Laplace transform' J. Comput. Physics vol. 33 no. 2 (1979) pp. 1-32.

8. Eggermont, P.P.B., 'Maximum penalized likelihood estimation and smooth EM algorithms for positive integral equations of the first kind', J. Numer. Funct. Anal. and Optimiz. Vol. 7, No. 7 (1996), pp. 737-754.

9. Engl, H.W. et al., 'Convergence rates for Tikhonov regularization of nonlinear ill-posed problems', Inverse Problems Vol. 5 (1989), pp. 23-40.

10. Essah, W.A. and Delves, L.M. 'On the numerical inversion of the Laplace transform' Inverse problems 4 (1988) pp. 705-724.

11. Gelfat, V.I. et al., 'Programs for signal recovery from noisy data using the maximum likelihood principle', Computer Physics Communications. Vol. 7 (1993), pp. 335-348.

12. Groetsch, C.W., 'The Theory of Tikhonov Regularization for Fredholm Equations of the First Kind', 1984 Pitman London.

13. Hofmann, B., 'Regularization for Applied Inverse and Ill-posed Problems', A Numerical Approach (Leipzig, Teuber), 1986.

14. Istratov, A.A. and Vyvneko, O.F., 'Exponential analysis in physical phenomenon', Published in Journal Review of Scientific Instruments, Vol. 10, No. 2 (1998), pp. 1233-1257.

15. Klein, G., 'On spline functions and statistical regularization of ill-posed problems', J. Comput. App. Math. Vol. 5, No. 4 (1979), pp. 259-263.

16. Lavrent'ev, M.M. et al., 'Ill-posed problems of Mathematical Physics and Analysis' (1986) Providence RI: American Math. Society.

17. Mead, L.R. et al., 'Maximum entropy in the problem of moments', J. Math. Physics Vol. 25, No. 8 (1984), pp. 2404-2417.

18. Medgyessy, P., 'Decomposition of Superposition of Density Functions and Discrete Distributions', Adam Hilger, Bristol, England (1977). 
19. Natterer, F., 'Error bounds for Tikhonov regularization in Hilbert spaces', J. Applied Analysis, Vol. 18 (1984), pp. 29-37.

20. Piessens, R. 'Laplace transform inversion' J. Comp. Appl. Maths. vol. 1 (1975) p. 115, vol. 2(1976), p. 225.

21. Piessens, R. and Branders, M. 'Numerical inversion of the Laplace transform using generalized Laguerre polynomials,' Proc. IEE 118 (1971) pp. $1517-1522$.

22. Pinkus, A. et al., 'Fourier Series and Integral Transforms', Cambridge University Press, Cambridge (1997), pp. 98-112.

23. Shan Hua Ning, 'A method for the numerical inversion of Laplace transform', Numer. Method. Comput. Math. Vol. 20, No. 3 (1999), pp. $231-236$.

24. Stoer, J. and Bulirsch, R., 'Introduction to Numerical Analysis', Springer Verlag (1978).

25. Talbot, A., 'The accurate numerical inversion of Laplace transform', J. Inst. Maths. Applics. Vol. 23 (1979), pp. 97-120.

26. Thompson, A.M., Brown, J.C., Kay, J.W. and Titterington, D.M., 'A comparison of Methods of choosing the smoothing parameter in image restoration by regularization', IEEE Trans. Pattern Anal. Machine Intell. Vol. 13(1991), pp. 326-339.

27. Thompson, A.M. and Kay, J., 'On some Bayesian choices of regularization parameter in image restoration', Inverse Problems Vol. 9(1993), pp. 749-761.

28. Tikhonov, A.N. and Arsenin, V.Y., 'Solution of ill-posed problems', Translated from the Russian. John Wiley, New York (1977).

29. Tikhonov, A.N., Leonov, A.S. and Yagola, A.G., 'Nonlinear = ill-posed Problems', Vols. 1, 2, Chapman and Hall, London (1998). 
30. Turchin, V.F., 'Solution of the Fredholm integral equations of the first kind in a statistical ensemble of smooth functions', USSR CMMP, Vol. 7 (1967), pp. 79-96.

31. Varah, J.M., 'Pitfalls in the numerical solutions of linear ill-posed problems', SIAM. J. Sci. Statist. Comput. Vol. 4, No. 2(1983), pp. 164-176.

32. Vasin, V.V. and Ageev, A.L., 'Ill-posed Problems With a priori Information', (1995), (Utrecht; VSP).

33. Wahba, G., 'Practical approximate solutions to linear operator equations, when data are noisy', SIAM. J. Numer. Anal. Vol. 14(1977), pp. 651677.

34. Whittle, P, 'Some results in time series analysis', Skand. Actuarietidekt. Vol. 35(1952), pp. 48-60.

35. Yamanoto, M., 'On ill-posedness and Tikhonov regularization for a multidimensional inverse hyperbolic problem', J. Math. Kyoto-Univ. Vol. 36(1996), pp. 825-856.

Received: June 11, 2005 